\title{
Designing Graphical User Interface (GUI) for Adjustable Robust Maximum Flow Problem
}

\author{
Diah Chaerani, Naufal Badruzzaman, Elis Hertini and Endang Rusyaman \\ Department of Mathematics, Faculty of Mathematics and Natural Sciences, \\ Universitas Padjadjaran \\ Jl. Raya Bandung Sumedang KM 21 Jatinangor Sumedang 45363 \\ e-mail: nbadruzzaman@gmail.com,d.chaerani@unpad.ac.id, elis.hertini@unpad.ac.id, \\ rusyaman@unpad.ac.id
}

\begin{abstract}
Maximum flow problem is one of optimization problems which aims to find the maximum flow value that is traversed in a network system. This problem can be solved using existing algorithms and linear programming. In the case of maximum flow, often the parameters used vary due to certain factors. [1] designed the Robust Counterpart Optimization Model for Maximum Flow Problems by assuming side and flow capacities from an indefinite point to destination point to solve the maximum flow problem with uncertainty. To facilitate the search for solutions with large amounts of data, a Graphical User Interface (GUI) was made. GUI is a pictorial interface of a program that can facilitate its users in completing their work such as counting, making, and so on. In this study, the GUI was created using Maple software and used the Adjustable Robust Counterpart Optimization Model made by [1]. Thus, the search for solutions to maximum flow problems can be resolved quickly and efficiently only by entering the data needed for calculations in the GUI. Keywords: Maximum Flow Problem, Robust Optimization, Adjustable Robust Counterpart, Graphical User Interface, Maple
\end{abstract}

\section{INTRODUCTION}

Maximum flow model is a graph application where the model can be used to find the maximum flow value that is passed in a network system. On each side there is a capacity that limits the amount of current that can be passed on each side. Examples of maximum flow problems that can be found include electricity, clean water, railroad tracks and so on. The Maximum Flow Problem was first introduced by Harris and Ross in 1954. [9] The study discussed the distribution by train in which every connected city has capacity. In reality, determining maximum flow of a network, there are uncertainty factors that affect the system, namely the flow capacity of each side that can change. For this reason, we need an optimization technique that can solve problems with these uncertainties.

One optimization method that can consider uncertainty is Robust Optimization. Robust optimization is an optimization method introduced by [4]. In Robust Optimization, the problem is solved by calculating the uncertainties in what data on a set of uncertainties.

2000 Mathematics Subject Classification: $90 \mathrm{C} 17$

Submitted: 2020-11-10, Revisions:2021-04-13, Accepted: 2021-08-01 
Adjustable Robust Optimization is introduced by [6] which can solve the problem of uncertainty on variables, which is by considering two sets of variables, namely the first set must be determined before resolving the uncertainty and the other can be calculated after the uncertainty is resolved. In the maximum flow problem, if the decision variable can be adjusted with uncertain parameters or can change, then the maximum flow problem can be solved by Optimizing Adjustable Robust Counterpart.

In the case of Operations Research problems, Maple software can be used as an interactive tool that can assist in conducting numerical experiments of Operations Research problems. Referring to [12], the solution of various Operational Research problems can be very easy by using packages and combining several Maple commands.

Besides being able to be used for calculations, we also need a calculation tool that is userfriendly and easy to use. Therefore, an interface or Graphical User Interface (GUI) was made to make it easy to solve optimization problems simply and easily to use. Maple has a Graphical User Interface application called Maplets. Maplets can make it easier for users to use software that has been developed. [11] mentioned that Maple software can provide an opportunity for users to develop images and graphics-based interfaces into deep mathematical solving programs. This research discusses the application of robust optimization to the problem of indeterminate maximum flow with the Adjustable Robust Counterpart method by considering uncertainty in the parameters and decision variables. Solution to the problem is presented in the form of Graphical User Interface (GUI) using Maple software.

\section{Methods}

2.1. Linear Optimization Model Maximum Flow Problems. Referring to [3], let $G=$ $(V, E)$ is a directed graph with the set of vertices $V$ and a set of arcs $E$. View in a network has $m$ vertices and $n$ arcs. On that network, state $x_{i j}$ is the flow from node $i$ to node $j$ above arc $(i, j)$ has a lower limit $l_{i j}=0$ and an upper limit $k_{i j}$. Let $x$ be the amount of flow on a network from the source node $s$ to the destination node $t$ The maximum flow model can be formulated generally as follows.

$$
\begin{aligned}
\max & x_{s t} \\
\text { s.t } & \sum_{j \in V} x_{i j}-\sum_{k \in V} x_{k i}-x_{s t}=0 \\
& \sum_{j \in V} x_{i j}-\sum_{k \in V} x_{k i}+x_{s t}=0 \\
& \sum_{j \in V} x_{i j}-\sum_{k \in V} x_{k i}=0, \forall i \in V-\{s, t\} \\
& 0 \leq x_{i j} \leq k_{i j}, \quad \forall(i, j) \in E
\end{aligned}
$$

Problem (1) can be written in the following form.

$$
\begin{aligned}
\max & x_{s t} \\
\text { s.t } & A x=0 \\
& 0 \leq x_{i j} \leq k_{i j}, \quad \forall(i, j) \in E
\end{aligned}
$$

2.2. Robust Optimization. Referring to [5], Robust optimization is a method for solving optimization problems where there is uncertainty in the data and is only known in a set of uncertainties. The general form of an uncertain linear optimization problem can be defined as follows.

$$
\begin{aligned}
\max & c^{T} x \\
\text { s.t } & A x \leq 0 \\
& \forall(x, A, b) \in \mathcal{U}
\end{aligned}
$$


where $c \in R^{n}$ is the objective function coefficient, $A \in R^{m \times n}$ is the constraint function coefficient matrix, and $b \in R^{m}$ is the right vertex vector.

There are three basic assumptions of "environmental decision-making" on the optimization Robust, that all decision variables representing the decisions "here and now",decision makers are fully responsible for the decisions taken if and only if the data has actually been defined in the set of uncertainties $\mathcal{U}$ in linear programming with uncertainty has constraints hard. Beside that, referring to [8] there are basic assumptions in Robust Optimization. First, the objective function $c^{T} x$ is certainly. Second, right-hand vector $b$ is certainly. Third, robustness to $\mathcal{U}$ can be formulated as problems that constrain-wise and uncertainty set $\mathcal{U}$ is a set that is closed and convex.

Referring to [8], assume that $c \in R^{n}$ and $b \in R^{m}$ are certainty value, then robust counterpart formulation of (3) can be defined as

$$
\begin{aligned}
\max & c^{T} x \\
\text { s.t } & a(\zeta) \leq 0 \\
& \forall \zeta \in \mathcal{Z}
\end{aligned}
$$

Note the constraints with uncertainty in (4), defined uncertainty parameters $a(\zeta)=\bar{a}+P \zeta$ where $\bar{a} \in R^{n}$ is a nominal value vector and $P \in R^{n \times L}$ is a confounding matrix. The set $\mathcal{U}$ can be defined as

$$
\mathcal{U}=\{a \mid a=\bar{a}+P \zeta, \zeta \in \mathcal{Z}\}
$$

where $\mathcal{Z} \in R^{L}$ is uncertainty sets of primitive factors. thus obtained

$$
(\bar{a}+P \zeta)^{T} x \leq b, \forall \zeta \in \mathcal{Z}
$$

The solution of Robust Optimization is called optimal robust. Furthermore, to reformulate the set of uncertainties $\mathcal{U}$ into a computational tractable problem, the following theorem applies.

Teorema 2.1. Assume that the set of uncertainty $\mathcal{U}$ in Equation (3) is an affine image of the limited set $\mathcal{Z}=\zeta \subset R^{n}$ and $\mathcal{Z}$ given as

(1) Systems of linear is inequality constraints $P \zeta \leq p$

(2) System of Inequality is Conic Quadratic $\left\|P_{i} \zeta-p_{i}\right\|_{2} \leq q_{i}^{T} \zeta-r_{i}, i=1, \ldots, M$

(3) System of Inequality is Linear Matrix $P_{0}+\sum_{i=1}^{\operatorname{dim} \zeta} \zeta_{i} P_{i} \geq 0$

In cases 2 and 3 , it is assumed also that the system of constraints that defined $\mathcal{U}$ are strictly feasible. Then the robust counterpart of linear programming with the uncertainty of (3) is equivalent to

(1) The linear programming problem in case 1.

(2) The quadratic conic problem in case 2.

(3) The semi definite problem for the case 3.

Computationally tractable robust counterpart representations of indeterminate linear problems for various classes of uncertainty sets are given in Table 1

TABLE 1. Formulation tractable to constraints with the set of uncertainties

\begin{tabular}{|c|c|c|c|}
\hline Uncertainty Sets & $\mathcal{Z}$ & Robust Counterpart & Tractability \\
\hline Box & $\|\zeta\|_{\infty} \leq 1$ & $a^{T} x+\left\|P^{T} x\right\|_{1} \leq d$ & LP \\
\hline Ellipsoidal & $\|\zeta\|_{2} \leq 1$ & $a^{T} x+\left\|P^{T} x\right\|_{2} \leq d$ & CQP \\
\hline Polyhedral & $D \zeta+q \geq 0$ & $\left\{\begin{array}{c}a^{T} x+q^{T} w \leq d \\
D^{T} w=-P^{T} x \\
w \geq 0\end{array}\right.$ & LP \\
\hline
\end{tabular}


2.3. Robust Counterpart for Maximum Flow Problems with the Box Uncertainty Set. Referring to [7], assume that a set of uncertainty $\mathcal{U}$ is a box. The set of uncertainty $\mathcal{U}$ can be defined as

$$
\mathcal{U}=\{k: l \leq k \leq u\}
$$

where $l$ and $u$ are two vectors in $R^{E}$ with $l \leq u$. So, Equation (2) can be written as follows.

$$
\begin{aligned}
\max & x_{t s} \\
\text { s.t } & A x=0 \\
& 0 \leq x \leq l
\end{aligned}
$$

This implies that the Robust Counterpart for the maximum flow problem with the box uncertainty set is a normal maximum flow problem with the $k$ side capacity vector replaced with $l$.

2.4. Robust Counterpart for Maximum Flow Problems with the Ellipsoidal Uncertainty Set. Referring to [7], assume that a set of uncertainty $\mathcal{U}$ is a ellipsoidal. The set of uncertainty $\mathcal{U}$ can be defined as

$$
\mathcal{U}=\left\{k: k=\bar{k}+P^{T} \zeta,\|\zeta\|_{2} \leq 1\right\}
$$

Thus, the Robust Counterpart Optimization model for the maximum flow problem with an ellipsoidal uncertainty set is obtained as follows.

$$
\begin{aligned}
\max & x_{t s} \\
\text { s.t } & A x=0 \\
& 0 \leq x \leq \bar{k}-\left\|P^{T}\right\|_{2}
\end{aligned}
$$

2.5. Robust Counterpart for Maximum Flow Problems with the Polyhedral Uncertainty Set. Referring to [1], assume that a set of uncertainty $\mathcal{U}$ is a polyhedral. The set of uncertainty $\mathcal{U}$ can be defined as

$$
\mathcal{U}=\left\{k: k=\bar{k}+P^{T} \zeta, d-D \zeta \geq 0\right\}
$$

Thus, the Robust Counterpart Optimization model for the maximum flow problem with an polyhedral uncertainty set is obtained as follows.

$$
\begin{aligned}
\max & x_{t s} \\
\text { s.t } & A x=0 \\
& x-\bar{k}^{T} \omega-d^{T} y \leq 0 \\
& D^{T} y=P^{T} \omega \quad x, y \geq 0
\end{aligned}
$$

2.6. Adjustable Robust Counterpart Optimization. Referring to [8] in multistage optimization, decision variables that are "here and now" can be relaxed. some decision variables can be adjusted at a later stage in accordance with the decision rule which is a function of some or all uncertain data. Adjustable Robust Counterpart in multistage optimization problems can be stated as follows.

$$
\min _{x, y}\left\{c^{T} x A(\zeta) x+B y(\zeta) \leq d \quad \forall \zeta \in \mathcal{Z}\right\}
$$

where $x \in R^{n}$ is the first stage, the "here and now" decision made before $\zeta \in R^{L}$ is realized. $y \in R^{L}$ states the decision "wait and see" and $B \in R^{m \times k}$ states the certain coefficient matrix. In practice, the $y(\zeta)$ function is often approached by affine or linear decision rules $y(\zeta)=y^{0}+Q \zeta$ where $y^{0} \in R^{k}$ and $Q \in R^{k \times L}$ is the coefficient in the decision rule to be optimized. So that the reformulation of equation (13) becomes as follows.

$$
\min _{x, y^{0}, Q}\left\{c^{T} x A(\zeta) x+B y^{0}+B Q \zeta \leq d \quad \forall \zeta \in \mathcal{Z}\right\}
$$


2.7. Adjustable Robust Counterpart Optimization for Maximum Flow Problems with Box Uncertainty Sets. Referring to [1], assume that parameters and indeterminate decision variables in the Adjustable Robust Counterpart model for the maximum flow problem are in the set of box uncertainty. So, we will get an Adjustable Robust Counterpart Model for Maximum Flow Problems with the set of box uncertainty as follows.

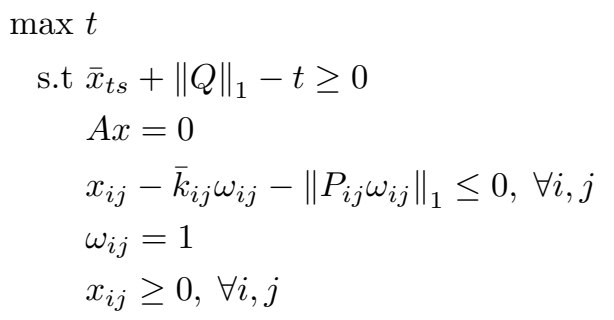

2.8. Adjustable Robust Counterpart Optimization for Maximum Flow Problems with Ellipsoidal Uncertainty Sets. Referring to [1], assume that parameters and indeterminate decision variables in the Adjustable Robust Counterpart model for the maximum flow problem are in the set of ellipsoidal uncertainty. So, we will get an Adjustable Robust Counterpart Model for Maximum Flow Problems with the set of ellipsoidal uncertainty as follows.

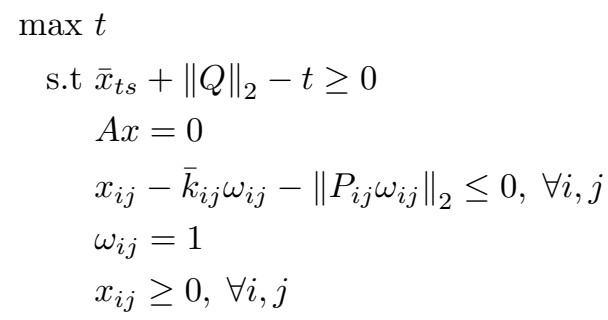

2.9. Adjustable Robust Counterpart Optimization for Maximum Flow Problems with Polyhedral Uncertainty Sets. Referring to [1], assume that parameters and indeterminate decision variables in the Adjustable Robust Counterpart model for the maximum flow problem are in the set of polyhedral uncertainty. So, we will get an Adjustable Robust Counterpart Model for Maximum Flow Problems with the set of polyhedral uncertainty as follows.

$$
\begin{array}{ll}
\max & t \\
\text { s.t } & \bar{x}_{t s}+d_{x}^{T} y_{x}-t \geq 0 \\
& A x=0 \\
& x_{i j}-\bar{k}_{i j} \omega_{i j}-d_{x}^{T} y_{x} \leq 0, \forall i, j \\
& D_{k}^{T} y_{k}=P_{i j}^{T} \omega_{i j} \\
& D_{x}^{T} y_{x}=Q \\
& \omega_{i j}=1 \\
& y_{k}, y_{x} \geq 0 \\
& x_{i j} \geq 0, \forall i, j
\end{array}
$$

2.10. Design of Graphical User Interface. Referring to [2], a Graphical User Interface (GUI) is a pictorial interface of a program. A good GUI is one that can make programs easier to use with a consistent look and with intuitive controls.

The main function of the designed GUI is to determine the optimal solution of the Maximum Flow Problem Optimization Model. The Maplet that is designed can determine the optimal solution from: 
(1) Optimization Model for Maximum Flow Problems with the objective function maximizing the flow from the source point to the destination point.

(2) Robust Optimization Model for Maximum Flow Problems with the objective function maximizing the flow from the source point to the destination point with the set of box uncertainty, ellipsoidal uncertainty, and polyhedral uncertainty.

(3) Adjustable Robust Counterpart Optimization Model for Maximum Flow Problems with the objective function maximizing the flow from the source point to the destination point with the set of box uncertainty, ellipsoidal uncertainty, and polyhedral uncertainty.

\section{Result And Discussion}

3.1. Data Preparation. The data used in this study is the data in the study of [10]. This research data is used to test the program by comparing the results of workmanship manually with the application. There is a pipeline system in Mumbai to supply water in various regions of Mumbai. The pipe between the two areas has a capacity expressed in gallons per hour. The water is supplied from source area A to destination area I through 7 other areas before reaching destination point I. Pipeline capacity between the two cities is presented in Table 2.

TABLE 2. Capacity of each pipe

\begin{tabular}{|c|c|c|}
\hline Source Area & Destination Area & Capacity (gallon/hour) \\
\hline A & B & 34 \\
\hline A & C & 20 \\
\hline A & E & 35 \\
\hline B & D & 20 \\
\hline B & E & 8 \\
\hline C & E & 8 \\
\hline C & F & 15 \\
\hline D & F & 10 \\
\hline D & G & 22 \\
\hline D & H & 18 \\
\hline E & D & 35 \\
\hline E & F & 15 \\
\hline F & H & 35 \\
\hline G & I & 48 \\
\hline H & G & 20 \\
\hline H & I & 30 \\
\hline
\end{tabular}

3.2. Results of Graphical User Interface Design. Graphical User Interface for Robust Counterpart Optimization Model for Maximum Flow Problems is an application program to make it easier for researchers to find the optimal solution from it.

The Maplet application is defined using the commands in the Maplets [Elements] package, then displayed using the Maplets [Display] command. The initial design of this user interface by arranging frames to enter data and display the results of calculations. Table 3 shows some of the elements used to create Maplets.

After the process of entering data, the user can process the data by pressing the buttons contained in the initial display frame and the system will process the input using proceduresexisting procedures. There are three windows that function to display the calculation results. In the Maplet main window there is a data input box and buttons for performing calculations. 
TABLE 3. Element in making Maplets

\begin{tabular}{|l|l|}
\hline \multicolumn{1}{|c|}{ Element } & \multicolumn{1}{c|}{ Function } \\
\hline TextField & Defines the text field for input in the Maplet application \\
\hline TextBox & Defines the text box to display output in the Maplet application \\
\hline Button & Defines buttons in the Maplet application \\
\hline Evaluate & Runs the maple procedure with the set of arguments given in the maple session \\
\hline BoxLayout & Defines the box layout in the Maplet application \\
\hline BoxColumn & Defines column boxes in the Maplet application \\
\hline BoxRow & Defines the box rows in the Maplet application \\
\hline Plotter & Defines the plot viewer in the Maplet application \\
\hline
\end{tabular}

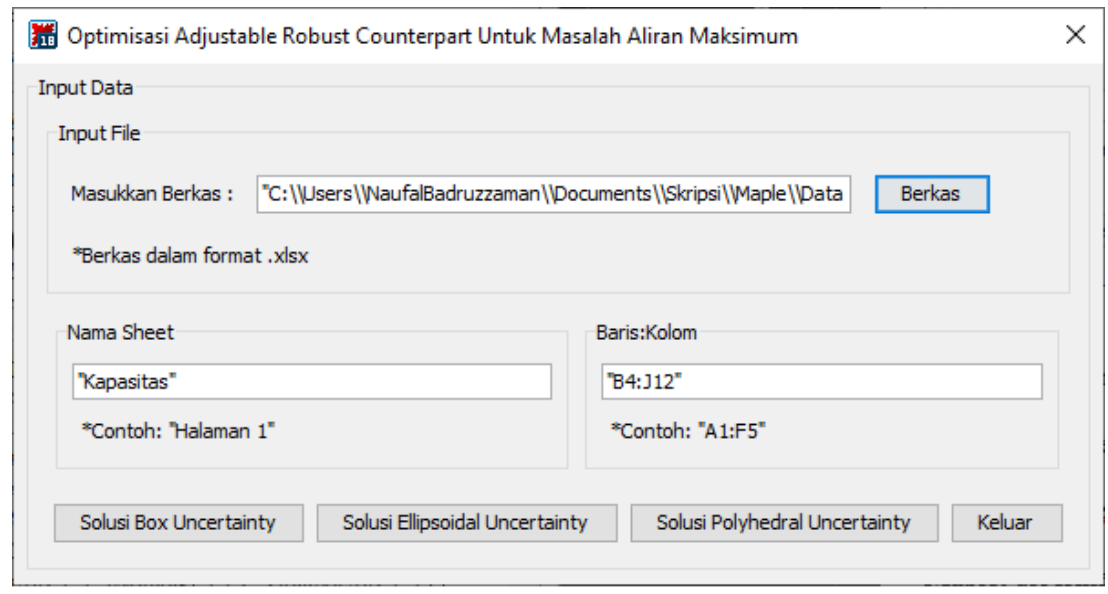

FiguRE 1. Maplet main window

When the "Solusi Box Uncertainty" button in the main window is pressed, it will display a window in Figure. The calculation process will work by entering the amount of disturbance then pressing the "Solusi" button.

Then when the "Solusi Ellipsoidal Uncertainty" button in the main window is pressed, it will display a window in Figure. The calculation process will work by entering the amount of the disturbance then pressing the "Solusi" button.

Then when the "Solusi Polyhedral Uncertainty" button in the main window is pressed, it will display a window in Figure. The calculation process will work by entering the amount of the disturbance then pressing the "Solusi" button.

3.3. Analysis of Results. This section discusses the comparison between manual calculations with Maple and calculations with a Graphical User Interface in the form of a Maplet that has been designed. The results of comparative calculations are shown in Table

Based on the test results, it can be seen that the optimal solution produced between manual work and Maplets are the same. Broadly speaking, it can be concluded that the Graphical User Interface that has been designed can facilitate users in finding optimal solutions from the Robust Counterpart Optimization Model for Maximum Flow Problems.

Based on the results of numerical experiments, the Maximum Flow Problem using Robust Optimization and Adjustable Robust Counterpart Optimization with a set of box uncertainty, ellipsoidal uncertainty, and polyhedral uncertainty produces a goal function value that is greater than the objective function value generated from the Jain and Garg (2012) models. This 


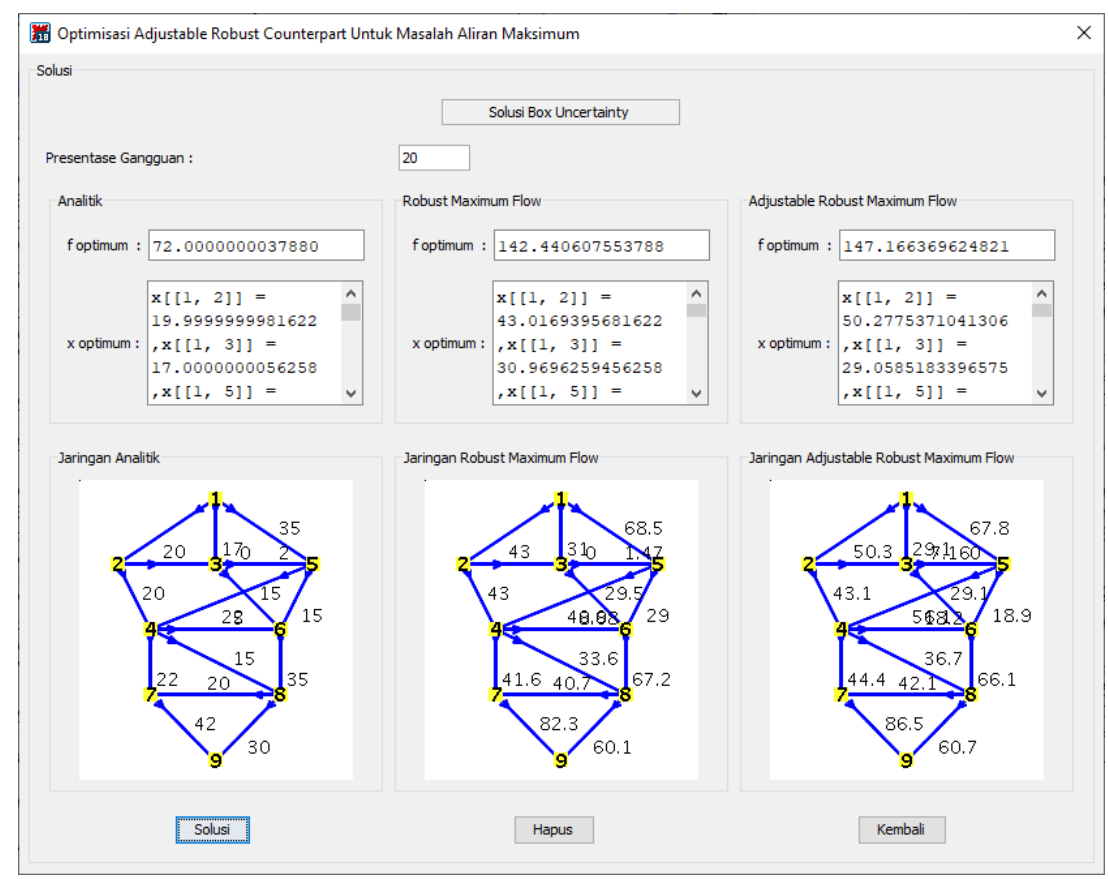

FIGURE 2. Box uncertainty solution window

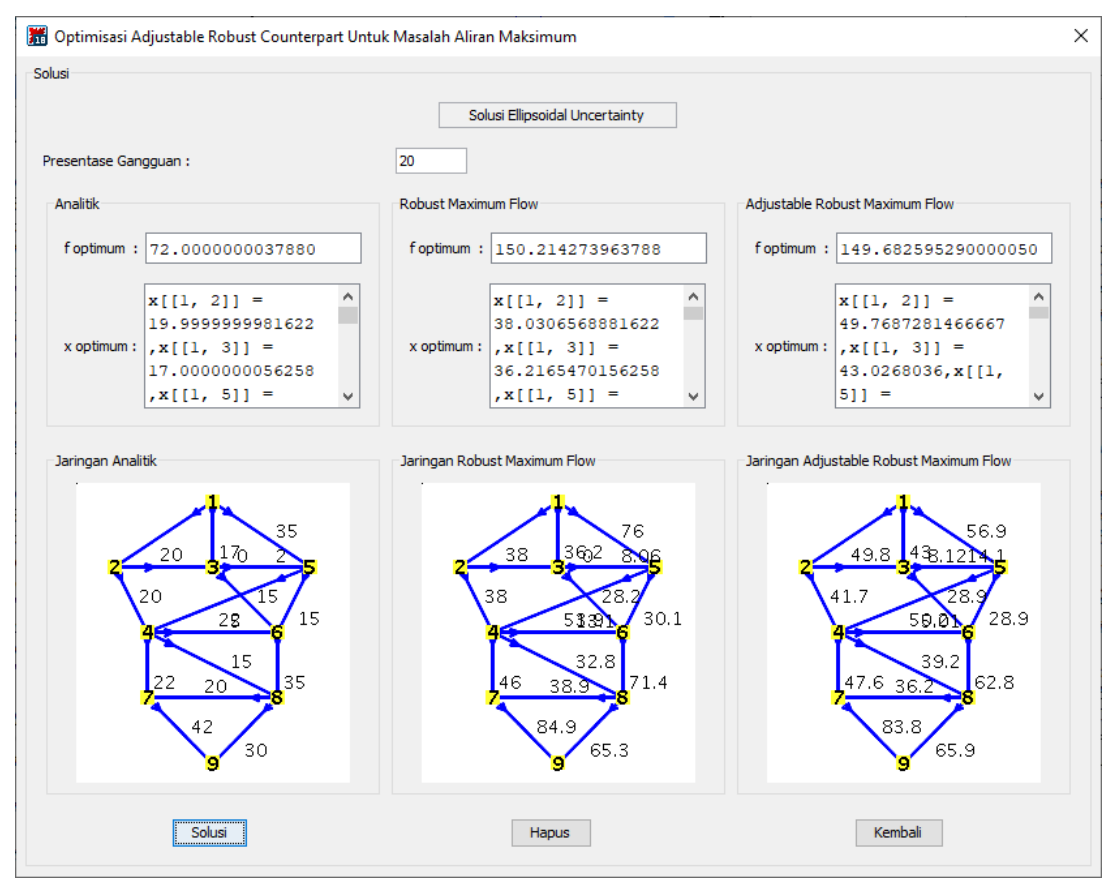

FIGURE 3. Ellipsoidal uncertainty solution window

happens because the pipe capacity between the two cities and the destination function is assumed to be indeterminate. 


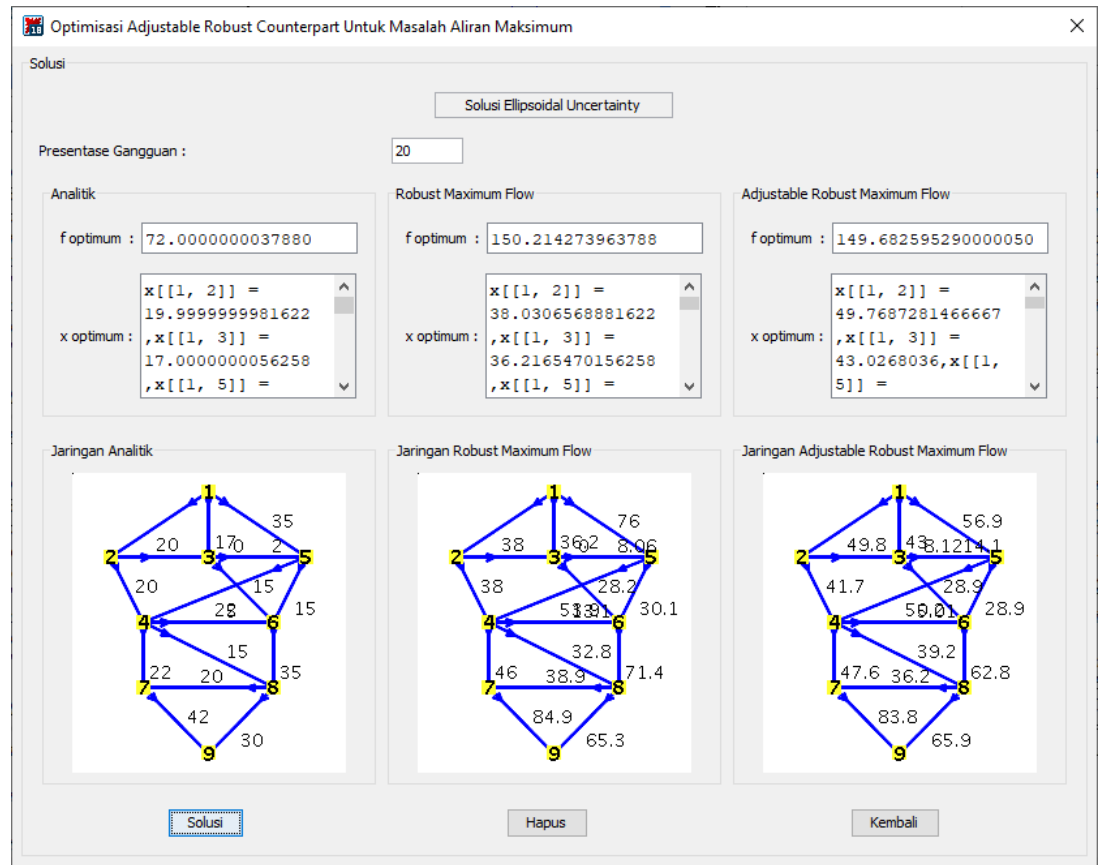

Figure 4. Polyhedral uncertainty solution window

TABLE 4. The comparison between manual calculations with Maple and Maplet GUI

\begin{tabular}{|c|c|c|c|c|c|c|c|c|}
\hline & \multirow{2}{*}{ Maple } & \multirow{2}{*}{ Maplet } & \multicolumn{2}{|c|}{ Box Uncertainty } & \multirow{2}{*}{$\begin{array}{l}\text { Ellipsoidal } \\
\text { tainty } \\
\text { Maple }\end{array}$} & \multirow{2}{*}{$\begin{array}{l}\text { Uncer- } \\
\text { Maplet }\end{array}$} & \multirow{2}{*}{$\begin{array}{l}\text { Polyhedral } \\
\text { tainty } \\
\text { Maple }\end{array}$} & \multirow{2}{*}{$\begin{array}{l}\text { Uncer- } \\
\text { Maplet }\end{array}$} \\
\hline & & & Maple & Maplet & & & & \\
\hline $\begin{array}{l}\text { Robust } \\
\text { Counter- } \\
\text { part }\end{array}$ & & & 142.441 & 142.441 & 150.214 & 150.214 & 143.426 & 143.426 \\
\hline $\begin{array}{l}\text { Adjustable } \\
\text { Robust } \\
\text { Counter- } \\
\text { part }\end{array}$ & & & 147.166 & 147.166 & 149.683 & 149.683 & 124.714 & 124.714 \\
\hline $\begin{array}{l}\text { Model } \\
\text { Nominal }\end{array}$ & 72 & 72 & & & & & & \\
\hline
\end{tabular}

\section{Conclusions}

Based on test results, manual calculations and calculations using Maplets for the Robust Counterpart Optimization Model for Maximum Flow Problems with a set of box polyhedral, ellipsoidal polyhedral, and polyhedral uncertainty produce the same solution, so that the Maplet application that has been designed can facilitate the user in determining optimal solution from the Robust Counterpart Optimization Model for Maximum Flow Problems.

\section{ACKNOWLEDGMENT}

This research is supported by Penelitian Dasar Kemenristek Dikti for the year of 2021, with contract No. 9/E1/KP/PTNBH/2021 and No. 1207/UN6.3.1/PT/2021 


\section{REFERENCES}

[1] Agustini, R. A., Chaerani, D., Hertini, E. 2020. Adjustable Robust Counterpart Optimization Model for Maximum Flow Problems with Box Uncertainty, World Scientific News, Volume 141, Pages 91 - 102.

[2] Ashi, R. Y. A., Ameri, A. A. 2011. Introduction to Graphical User Interface (GUI) MATLAB 6.5, IEEE UAEU Student Branch.

[3] Bazaraa, M. S., Jarvis, J. J., Sherali, H. D. 2010. Linear Programming and Network Flows (4th ed.), Hoboken, New Jersey, John Wiley Sons, Inc.

[4] Ben-Tal, A., Nemirovski, A. 1999. Robust Solutions of Uncertain Linear Programs, Operations Research Letters, Volume 25, Pages 1 - 13.

[5] Ben-Tal, A., Nemirovski, A. 2002. Robust Optimization - Methodology and Applications, Mathematical Programming, Volume 92, Pages 453 - 480.

[6] Ben-Tal, A., Guslitzer, E., Nemirovski, A. 2004. Adjustable Robust Solutions of Uncertain Linear Programs, Mathematical Programming, Volume 99, Pages 351 - 376.

[7] Chaerani, D., Roos, C. 2006. Modelling Some Robust Design Problems via Conic Optimization, Operation Research Proceedings 2006, Pages 209 - 214.

[8] Gorissen, B. L., Yanikoglu, I., Hertog, D. D. 2015. A Practical Guide to Robust Optimization, Omega: The International Journal of Management Science, Volume 53, Pages 124 - 137.

[9] Harris, T. E., Ross, F. S. 1955. Fundamentals of a Method for Evaluating Rail Net Capacities, Research Memorandum.

[10] Jain,C., Garg, D. 2012. Improved Edmond Karps Algorithm for Network Flow Problem, International Journal of Computer Applications, Volume 37.

[11] Narayanan, A. and Narayanan, G., 2010, June. On Maplet development and programming tutorial for science and engineering students. In 2010 Annual Conference Exposition (pp. 15-921).

[12] Parlar, M. 2000. Interactive Operations Research with Maple: Methods and Models, Hamilton: DeGroote School of Business. 